\section{IS PRISCILLA, THE TRAPPED POSITRON, AN INDIVIDUAL? QUANTUM PHYSICS, THE USE OF NAMES, AND INDIVIDUATION}

\author{
Décio Krause \\ Universidade Federal de Santa Catarina \\ Brasil
}

\begin{abstract}
RESUMEN: El Premio Nobel Hans Dehmelt aprisionó un positron, que fue llamado "Priscilla". Dehmelt dijo que no podemos dudar de la identidad de Priscilla. Eso nos coloca un problema filosófico, puesto que aprendimos con la mecánica cuántica (en sus principales interpretaciones) que los objetos cuánticos no deberían tener individualidad. En este artículo, discutimos las cuestiones de nombrar los quanta y las cuestiones de la individuación. Conclúyenos que, a pesar de que Dehmelt nombró algo, ese algo no es un individuo.
\end{abstract}

PALABRAS CLAVE: Individuos; no-individuos; partículas aprisionadas; individualidad; identidad.

\section{INDIVIDUALS AND NON-INDIVIDUALS}

It is quite difficult to write down a precise definition of what would be an individual. Here, we are interested in using this term to refer to individual (physical) objects, in distinction to both mass and kind terms, leaving aside other possibilities. Informally, an individual is something which, even belonging to a swarm of entities of the same species, can be identified as being that individual, a numerically singular thing we can identify in other situations where we find it (put another way, it can be identified as being that object in all possible words). An individual can bear a name, a label, something that can be used to refer to it without confusion in whatever context. In general, we don't look for a "definition" of the concept, but take it as primitive and ask what confers an individual its individuality instead, looking for some Principle of Individuation. Philosophy has presented us a lot of different answers we will not revise here (see Quinton, 1973).

Just to sum up, we recall that most of the answers fall in one of the two general schemas, namely, the theories which admit a form of substratum and those which reject
¿ES PRISCILLA, EL POSITRÓN ATRAPADO, UN INDIVIDUO? LA FÍSICA CUÁNTICA, EL USO DE NOMBRES Y LA INDIVIDUACIÓN

ABSTRACT: Nobel laureate Hans Dehmelt trapped a positron for three months, which was named "Priscilla". Dehmelt said that we could not doubt the identity of Priscilla. This poses us a philosophical problem, for we have learnt from quantum physics (in most of its interpretations) that quantum objects shouldn't have individuality. In this paper, we address on the questions of naming quanta and the issue of individuation. We conclude by saying that, although Dehmelt has named something, this something is not an individual.

KEY WORDS: Individuals; non-individuals; identity; quantum objects; trapped particles.

this idea, the bundle theories. Saying in brief, according to the former, that what confers individuality to an individual is something over and above its qualities, or properties, while the last ones accept that an individual is to be identified with the collection its properties: it is a bundle of properties. Both views have problems: the first ones need to specify the nature of this substratum, while the last ones encounter difficulties with logic, for we may ask whether it can be possible for "two" individuals to have exactly the same set of properties. The well known answer given by Leibniz was expressed by his Principle of the Identity of Indiscernibles: if "two" entities have the same properties (are indiscernible), then they are the very same object, they are identical, and there are no "two" of them, but only one. Classical logic and standard mathematics incorporate this principle from one way or another, so that all theories found on such a ground are in principle Leibnizian in this sense. A wide discussion on these issues is given in French \& Krause, 2006.

Quantum physics has been the realm par excellence where non individual entities do appear. Let us use the expression non-individual to refer to a kind of entity that, although 
being able to participate as an element of a swarm of entities of the same species, has no numerical identity, cannot e identified by a label or by a name. Quantum objects, the typical case being those of bosons, are entities of this kind. Roughly speaking, they are so that if an object of this kind is substituted by another of the similar kind, nothing physically relevant will be detected (Bose-Einstein statistics). Note that the situation here is distinct, say, from that where we are observing an ant and then she enters in the ants' nest joining other ants of the same species, and we are no more able to identify it. But, in this case, we can for instance paint the ant with a small mark of ink, so that, even entering in the nest, when arising from it again we have the grounds for saying that that ant is "our" ant. Concerning elementary quantum objects, in a time when we still did not trap particles, it was claimed by Schrödinger that "we cannot mark an electron, we cannot paint it red" (Schrödinger, 1953). Quantum objects apparently are of a distinct nature from their "classical" twins. They would not have identity. Are there ontologically different? Have they identity, as Priscilla apparently has?

According to standard quantum mechanics, two quantum objects, when interacting, may enter in superstition, and then no identification is any more possible. Some people say that their individuality is lost. The story of this nonindividuality entered in physics when Planck, $n$ deriving his radiation law, admitted the distribution of indistinguishable non-individual quanta over oscillators. In 1926, Born, while defending the corpuscular as opposed to wave-like conception, acknowledged that these corpuscles could not be identified as individuals. In the same year, Heisenberg noted that Einstein's theory of the ideal quantum gas implies that the "individuality of the corpuscle is lost" (for historical details, see French \& Krause, 2006, 85ff). Weyl and Schrödinger were other important thinkers who defended the non-individuality of elementary "particles". Schrödinger was so inclined to deny the identification of these objects (elementary particles) that suggested that the concept of identity cannot be applied to them (French \& Krause, 2006).

In a series of works, we have pushed this idea that identity is meaningless to some entities to the development of logic-mathematical systems where expressions like $x=y$ (so as its negation, $\neg(x=y)$ ) are not a well formed formulas (where $x$ and $y$ are individual variables ranging over non-individuals) to higher order logics and to a theory of collections of these objects, termed quasi-set theory (see French \& Krause, 2006, for all the motivation, history and technical details). The reason for this choice was first to pursue Schrödinger's claims mentioned above and, further, to avoid that the principle of the identity of indiscernibles, write in the language of second order logic as $\forall x \forall y(\forall F$ $(F(x) \leftrightarrow F(y)) \rightarrow x=y)$, results to be a theorem of the considered logic. In fact, if among the properties of an object $a$ we consider that defined as $I_{a}(x)==_{\text {def }} x=a$, which we can call "to be identical to $a$ ", then if $b$ partake with $a$ all its properties (that is, if $a$ and $b$ are indiscernible), then it it has in particular that property, hence $b=a$ and there are no "two" objects, but only one, which can be referred to by either $a$ or $b$ (they turn to be identical, the same object).

Even if we consider extensional set theory, identity is defined so that $a=b$ if and only if they have the same elements and (if there are Urelemente), if and only if they belong to the same sets, that is, if they are indiscernible. First order logic cannot specify identity completely, in the sense that there exist non normal structures which are elementary equivalent to a normal model. A normal model is one whose the primitive predicate of identity is interpreted in the diagonal of the domain (the relation of identity of the model) -for details, see Mendelson, 1989, 100. Thus, we can say that non-individuals are those entities to which the predicate of identity is not defined, in particular, we don't have $a=a$ if $a$ is a non-individual. Hence, they fail to obey the classical theory of identity.

But, within standard mathematics (without loss of generality, we can assume that all we shall consider can be developed in the first order Zermelo-Fraenkel set theory encompassing the Axiom of Foundation, ZF), every object is an individual. In fact, take $a$ and $b$. Then we can always form the singleton $\{a\}$, and $x \in\{a\}$ if and only if $x=a$, that is, if and only if $x$ is identical to $a$. In extensional contexts, we can regard $x \in\{a\}$ as the definition of the predicate $I_{a}(x)$. Then, if $a$ and $b$ are distinct, it is false that $I_{\mathrm{a}}(b)$, so they are distinguished by at least one "property", and Leibniz's principle holds. The usual way of considering indiscernible objects within "classical" frameworks is to work with structures embedded in the well founded universe of ZF, called $V$. As it is well known, $V$, as a structure, is rigid, that is, its only automorphism is the identity 
function. But we can construct structures which are not rigid in this sense. Then, we say that two objects $a$ and $b$ of the domain of one of such structures are indiscernible (form the point of view of the structure) if and only if there is an automorphism $h$ of this structure such that $h(a)=b$. For example, 2 and -2 are indiscernible relatively to the

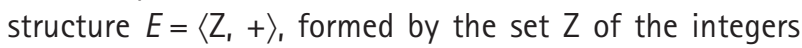
and the addition of integers, whose only automorphisms are the identity function and $h(x)=-x$.

This is more or less what happens in the formalism of quantum theory. In order to say that, say, two bosons are indiscernible, we take the join system as described by a symmetric vector (or a wavefunction) which is invariant by any exchanging of their coordinates. That is, they are invariant by permutations. These symmetry conditions are essential part of the quantum formalism. But, in $Z F$, we can prove that any structure (built in ZF) can be extended to a rigid structure. That is, by adding adequate objects, relations, and operations to the structure, we can obtain a rigid one; for instance, add to $E$ above all singletons of the elements of $Z$, getting $E^{\prime}=\left\langle Z_{1}+,\{0\},\{1\},\{-1\},\{2\}\right.$, $\{-2\}, \ldots\rangle$, which is rigid, as it is easy to see. In short, the way of considering indiscernible objects within standard mathematics (and logic) is by means of some trick: we take individuals first, identified by their names, coordinates -which is the same- or by descriptions, and then we introduce some device (like symmetry postulates) which makes them (artificially) indiscernible. But, let us emphasize, in the whole ZF they are individuals, and can always be identified as such. There is no way: as we said, classical logic and mathematics are Leibnizian.

These considerations have importance for the philosophical foundations of quantum theory. We can treat quantum objects as individuals, on a pair with their "classical" counterparts (described by classical physics), but at the expenses of restricting the states and set of observable available to them (French \& Redhead, 1998, French \& Krause, 2006). But, in this case, their individuality must be ascribed by some kind of substratum (French \& Redhead, 1998, French \& Krause, 2006), so we enter in the realm of substratum theories, and metaphysics becomes complicated. Bohm's theory, as it is well known, does that by assuming that quantum objects are individuals (like "classical particles"), but the dynamics has, beyond the Schrödinger equation, a "guiding equation", which depend on the coordinates of the particles (see Goldstein, 2006). This shows that the mentioned trick is still present: we have individuals in the start, but the formalism will provide the way of treating them as they were not or, as in this case, as if they have the typical properties of non-individuals. Why not to search for a right way of describing them "directly"?

These are of course interesting topics, but let us end this digression and go back to our point. Can we say that the claim that we can name a quantum object, put it in a jail, isolate it, makes this object an individual? Let us address to this question next.

\section{TRAPPING QUANTUM OBJECTS}

The Nobel laureate (1989) Hans Dehmelt has developed an important work in trapping quantum objects, something which nowadays has been used in quantum computation. In particular, Dehmelt trapped a positron (the anti-particle of the electron) by three months, and called it "Priscilla". He has also trapped other objects, like a barium ion which was named "Astrid", but we shall be fixed here in considering Priscilla. Dehmelt says: "[t]here can be little doubt about the identity of Priscilla during this period, since in ultrahigh vacuum she never had a chance to trade places with a passing antimatter twin. The well-defined identity of this elementary particles is something fundamentally new, which deserves to be recognized by being given a name, as pets are give names of persons" (Dehmelt, 1990 - see also Dehmelt, 1989, Heppenheimer, 1994). The technical details do not important us here, but just the fact that, according to Dehmelt, Priscilla has individuality, is an individual.

Why Priscilla would be an individual? The obvious answer goes in saying that we can point to a trap at Dehmelt's laboratory and say: "that's Priscilla, inside the trap". The fact that Priscilla has a name, a well located position in the laboratory, in a way that it cannot interact with other objects of the same kind, makes it an individual. At least it seems. But we need first to identify the trap, which we do by considering the various asymmetries there are in the laboratory. But let us suppose that Dehmelt receives the visit of a colleague from another university and that we showed Priscilla to him as described above: he comes close to the trap and says "That's Priscilla!". But suppose that 
Dehmelt is just discussing the philosophical point we are concerned here (it would be wonderful if he did it) with his students, and commanded them to cancel the experience (so eliminating the positron) and making it again, in order to discuss with them if the "new" trapped positron will have any difference to Priscilla. But the students, typically, resolve to do nothing: "Prof. Dehmelt will not perceive the difference between the two positrons", says one of them. Are they right? We think they are. There are no difference between positrons, except that one of then is here and now in a certain trap we are just looking at.

Thus, we may say that it is a certain spatio-temporal location that makes Priscilla an individual, being the only property we can ascribe to her which is not partaken by any other quantum object. But this is also not sufficient. Leaving aside the difficult problem of specifying the nature of the concepts of space and time in this assertion (say: are they those of Newtonian mechanics or are they relativist ones?), we can show the inadequacy of this proposal alternatively. In fact, suppose there are two quite similar traps in Dehmelt's laboratory (suppose this is possible without further consideration about the individuality of the traps, another difficult problem, as it is well known), both with a positron trapped in. Which one is Priscilla? Well, you can say: that one in the trap near the window. But suppose Dehmelt is absent from his laboratory for a moment, during which the students exchange the traps one another, and leave the light sufficiently weak so that Dehmelt doesn't perceive the new configuration of his laboratory. Then the students ask him: where is Priscilla? For sure Dehmelt will point to the trap near the window and say: "She's there!". Without a careful analysis of the trap (not the positron), he cannot distinguish between the two positrons. Only by distinguishing between the traps, by noticing that they were changed, Dehmelt will be able to say that Priscilla is not that positron, but the another one.

Furthermore, suppose that Dehmelt ended his experiment, which resulted in trapping Priscilla, not on March, 141975 (I don't know the exact date: mines are fictitious), but on April, 13 1975. Of course he could call Priscilla that positron of April, 13. Can we say that it would be any difference for the scientific community? Of course not, although we cannot prove that the positron of March, 14 and the positron of April, 13 are the very same object. This claim seems to have no sense at all. Positrons do not have individuality, they are not individuals (of course there are difficulties also concerning macroscopic objects, as Hume has emphasized, but we shall not touch this point here too).

\section{The USE OF nAMES}

Finally, let us address some remarks to the use of names in the quantum realm (for further details, see French \& Krause, 2006, chap.5 -see also Dalla Chiara, 1985, Toraldo di Francia, 1986). Dalla Chiara and Toraldo di Francia have claimed that microphysics "is the land of anonymity" (1993), where there are no proper names, and any identification we made of a quantum object provides it only a "mock individuality", having "very brief duration". Of course Dehmelt's experiences can be included in this description, if we relax the "brief duration" to three months. After that period, Priscilla cannot be identified any more (sometimes the object does not exist any more!), and can be remembered only by reference of being the object which some day was in that trap, and not by some peculiar property of itself, which does not make reference to this fact.

Concerning names, the philosophical literature distinguishes between two main uses of names in science: the nondescriptivist (or referential) use and the descriptivist use. A name, used in the non-descriptivist sense, just labels, or "tags", its bearer, having no other meaning than to stand for the object it names. Its meaning is entirely given by its reference. As famously put by Stuart Mill, a proper name has denotation, but not connotation. A kind term like "serpent", on the contrary, has both denotation, since it can be used to refer to a specific reptile, and connotation, for it may be used also to mean "danger" or "treason" (see ibid.). According to this view, names refer for they are attached to the object as a person's name is assigned to her at birth. Of course this use of names is not free from difficulties, we shall not discuss here (see French \& Krause, 2006, chap. 5). Thus, in a non-descriptivist view, "Priscilla" is just the name of that positron trapped by Dehmelt some time ago, and its meaning is its reference, given by ostension.

In the descriptivist use, names refer by virtue of the descriptive content associated with the name. This content is usually specified by a definite description satisfied by the object answering to that name. Thus, associated to "Pris- 
cilla", there is also a definite description like "the positron trapped in Dehmelt's laboratory". So, in order this kind of use of names work, we need to provide the grounds for showing that the description has being uniquely picked out by the object referred to. Let us be more specific on this point. Writing $P(x)$ for "to be a positron" and $D(x)$ for "is trapped in Dahmelt laboratory", the above description can be written $D\left({ }_{1 x P}(x)\right)$ in the usual notation of the theory of descriptions, which according to Russell can be translated to $\exists x(P(x) \wedge \forall y(P(y) \rightarrow y=x) \wedge D(x))$. Hence, we need to prove that Priscilla is the only positron trapped in Dehmelt's laboratory, and we cannot do that since we have not an adequate theory of identity for positrons -really, what means $y=x$ in what respect positrons, or other quantum objects? We hope that the above argumentation has settled this point, with the cases involving the exchange of traps, and with the possible repetition of the experiment.

How to understand "Priscilla" and Priscilla, trapped by Dehmelt? Priscilla is a quantum object, and as such, has its properties, or characteristics, prescribed by physical law. As Toraldo di Francia has put, it is a nomological object (1981, 222; Dalla Chiara and Toraldo di Francia, 1993). All positrons have the same prescribed characteristics, and a particular location of one of them is to be understood as something which gives it only a mock individuality, as referred to above. Despite being trapped by three months, the case of Priscilla is like that of two similar quantum objects which go apart to a sufficient distance to be distinguished (say in a Wilson Chamber), although what we observe in a Wilson Chamber are not the particles themselves, but drops of supersaturated water vapor arranged along definite tracks, which make us conclude that the tacks were formed by quantum objects passing at high speeds (Heisenberg, 1949, 4). But these objects, when sufficiently closer, enter in superposition and, even after a possible later depart, we cannot say anymore which is which. Al of this is well known from the technical point of view. The difficult task is to explain that, and hence we shall turn to the understanding of "Priscilla".

We think that we ought to distinguish between two languages we normally use in physics: let us call them the object language, which is the language of the formalism proper, in which we express the theory's concepts and principles, and the metalanguage, where we interpret the object language in order to make assertions about the facts described by the theory and communicate the results. The differences between these languages help us here, and something written by George Berkeley at $\$ 51$ of his $A$ Treatise Concerning Human Knowledge also applies here; as he said, "we ought to "think with the learned, and speak with the vulgar.". Our vulgar metalanguage encompasses names and all other resources we find necessary to a better understanding of the achieved results and to communicate them, while the language proper we use to think about quantum physics is purely mathematical. In this "learned", object language, there should be no names at all (at least according to a reasonable interpretation of quantum formalism which sees quantum objects as non-individuals). Thus, "Priscilla" makes part of the vulgar language, to use Berkeley's term, but cannot be incorporated to the formalism without making great and sometimes difficult restrictions. It is simply a word we use to tell to our friends that something was done in the laboratory, but cannot serve to provide the grounds that we have trapped an individual. Schrödinger himself has also addressed to this use of an "inadequate language" to express facts which are alien to the real state of affairs, when said that the language we normally use to speak about quantum objects, taken from classical physics, "constantly drives our mind to ask for information which has obviously no significance. Its imaginative structure exhibits features which are alien to the real particle" (Schrödinger, 1957, 204). After this argumentation, we hope the reader can for his/her own opinion. 


\section{REFERENCES}

Dalla Chiara, Maria Luisa (1985): "Names and Descriptions in Quantum Logic", in Mittelstaedt, Peter and E.-W. Stachow (eds.), Recent Developments in Quantum Logics, Mannheim: Bibliographisches Institut, pp. 189-202.

Dehmelt, Hans Georg (1989): Autobiography, at the Nobel Prize Organization web page.

Dehmelt, Hans Georg (1990): "Experiment on the Structure of an Individual Elementary Particle", Science, New Series, Vol. 247, No. 4942: 539-45.

French, Steven and Michael Redhead (1988): "Quantum Physics and the Identity of Indiscernibles", British Journal for the Philosophy of Science, 39: 233-246.

French, Steven and Décio Krause (2006): Identity in Physics: A Historical, Philosophical, and Formal Analysis, 0xford: Oxford University Press.

Recibido: 5 de junio de 2009

Aceptado: 10 de diciembre de 2009
Heisenberg, Werner (1949): The Physical Principles of Quantum Theory, New York: Dover.

Heppenheimer, Thomas A. (1994): "A Positron Named Priscilla: Trapping and Manipulating Atoms", in Bartusiak, Marcia et al. (eds.), A Positron Named Priscilla: Trapping and Manipulating Atoms, Washington, D.C.: National Academy Press, pp. 34-59.

Mendelson, Elliot (1987): Introduction to Mathematical Logic, London: Chapman \& Hall, 4th. ed.

Quinton, Anthony (1973), The Nature of Things, London: Routledge \& Kegan Paul.

Schrödinger, Erwin (1953): "What is matter?", Scientific American, Sept.: 5257.

Schrödinger, Erwin (1957): "What is an Elementary Particle?", in Schrödinger, Erwin, Science, Theory, and Man, London: Allen \& Unwin, pp. 193-223.

Toraldo di Francia, Giuliano (1981): The Investigation of the Physical World, Cambridge: Cambridge University Press. 